Revue musicale OICRM

\title{
Interstellar de Hans Zimmer : plongée musicale au coeur des drames humains, par-delà l'infiniment grand. Pour une autre approche de l'esthétique zimmerienne
}

\section{Chloé Huvet}

Volume 5, numéro 2, 2018

Création musicale et sonore dans les blockbusters de Remote Control

URI : https://id.erudit.org/iderudit/1054149ar

DOI : https://doi.org/10.7202/1054149ar

Aller au sommaire du numéro

\section{Éditeur(s)}

Observatoire interdisciplinaire de création et recherche en musique (OICRM)

ISSN

2368-7061 (numérique)

Découvrir la revue

Citer cet article

Huvet, C. (2018). Interstellar de Hans Zimmer : plongée musicale au coeur des drames humains, par-delà l'infiniment grand. Pour une autre approche de l'esthétique zimmerienne. Revue musicale OICRM, 5(2), 103-124.

https://doi.org/10.7202/1054149ar
Résumé de l'article

Le modèle souvent relayé dans la littérature dominante sur Hans Zimmer, mettant en valeur la masculinité et le caractère épique de la musique de Zimmer, le son massif et puissant de ses partitions, et l'omniprésence d'une pulsation rythmique marquée tend à délaisser tout un pan de l'approche zimmerienne de la composition cinématographique et s'avère peu adéquat pour analyser les partitions les plus récentes du compositeur qui ne correspondent pas à ce schème. C'est le cas, en particulier, d'Interstellar, dont la bande-son excède largement ce cadre général prédéfini.

Nous montrerons comment le traitement de la musique place l'humain au coeur du film, et de quelles manières Zimmer fait des liens entre Cooper et sa fille Murph le point de mire de sa partition. En filigrane, notre étude propose, à partir de l'exemple de ce film, une autre approche de l'esthétique du compositeur, qui se veut complémentaire au modèle dominant en invitant à repenser la filmographie de Zimmer - y compris son versant épique - sous un angle riche et ouvert. 


\title{
Interstellar de Hans Zimmer : plongée musicale au coeur des drames humains, par-delà l'infiniment grand. Pour une autre approche de l'esthétique zimmerienne
}

\author{
Chloé Huvet
}

\begin{abstract}
Résumé
Le modèle souvent relayé dans la littérature dominante sur Hans Zimmer, mettant en valeur la masculinité et le caractère épique de la musique de Zimmer, le son massif et puissant de ses partitions, et l'omniprésence d'une pulsation rythmique marquée tend à délaisser tout un pan de l'approche zimmerienne de la composition cinématographique et s'avère peu adéquat pour analyser les partitions les plus récentes du compositeur qui ne correspondent pas à ce schème. C'est le cas, en particulier, d'Interstellar, dont la bande-son excède largement ce cadre général prédéfini.

Nous montrerons comment le traitement de la musique place l'humain au cœur du film, et de quelles manières Zimmer fait des liens entre Cooper et sa fille Murph le point de mire de sa partition. En filigrane, notre étude propose, à partir de l'exemple de ce film, une autre approche de l'esthétique du compositeur, qui se veut complémentaire au modèle dominant en invitant à repenser la filmographie de Zimmer - y compris son versant épique - sous un angle riche et ouvert.
\end{abstract}

Mots clés : Interstellar; Hans Zimmer ; Christopher Nolan ; bande-son ; musique de film ; effets sonores ; immersion ; mixage.

\begin{abstract}
The model often relayed in the mainstream literature about Hans Zimmer, highlighting the masculinity and epic character of Zimmer's music, the massive and powerful sound of his scores, and the omnipresence of a marked rhythmic pulsation tends to neglect an important part of Zimmer's approach to film scoring, and proves to be inadequate for analyzing the composer's most recent scores that do not correspond to this schema. This is the case, in particular, of Interstellar, which soundtrack largely exceeds this predefined general framework.

I show how the treatment of the music places the human at the heart of the film, and in what ways Zimmer focuses his score on the relationship between Cooper and his daughter Murph. From the example of this film, my study also offers another approach of the composer's aesthetics, which is complementary to the dominant
\end{abstract}


model, inviting to rethink Zimmer's filmography - including its epic side - under a rich and open angle.

Keywords: Interstellar; Hans Zimmer; Christopher Nolan; soundtrack; film music; sound effects; immersion; mixing.

Longtemps délaissé par les cinémusicologues ${ }^{1}$, le compositeur Hans Zimmer fait, depuis quelques années, l'objet d'études et d'analyses approfondies, tant en ce qui a trait à son écriture, à son processus compositionnel qu'au mode de fonctionnement de son studio Remote Control Productions ${ }^{2}$. Parmi les publications les plus significatives, une analyse des partitions de Watchmen (Zack Snyder, 2009) et Inception est développée dans une perspective comparative par Aylish Wood (Wood 2013, p. 417-436), tandis qu'une étude entière consacrée à The Dark Knight : le chevalier noir de Christopher Nolan (2008) a été publiée dans la série des Film Score Guides (Hexel 2016). Par ailleurs, Benjamin Wright et Matt Hurwitz se penchent sur le processus compositionnel particulier mis en œuvre par Zimmer et les membres de son studio (Wright 2015, p. 319-327 ; Hurwitz [2007] 2012, p. 254-257). Enfin, au sein d'un ouvrage collectif consacré à la musique de film épique (Meyer 2016), Frank Lehman s'emploie à dégager les principales caractéristiques du style « masculin » du compositeur (Lehman 2016, p. 27-55), tandis que Joakim Tillman (Tillman 2016, p. 59-85) propose de mettre en lumière la narrativité musicale dans Gladiator (Ridley Scott, 2000).

Pour importantes qu'elles soient, la plupart de ces publications insistent surtout sur la masculinité et le caractère épique de la musique de Zimmer, le son massif et puissant de ses partitions, et l'omniprésence d'une pulsation rythmique marquée. Ce modèle dégagé par les chercheurs, dont on peut effectivement appliquer plusieurs éléments à nombre des partitions du compositeur - en particulier les trames musicales conçues pour les films d'action - génère l'illusion d'un langage musical relativement homogène chez Zimmer, que l'on retrouverait de film en film de manière quasi identique. Or, comme le souligne à juste titre Vasco Hexel, "Zimmer has over the years changed his musical vocabulary drastically and repeatedly. He points out the need to "re-invent" his style periodically because he is being copied so readily» (Hexel 2016, p. 7).

Le modèle souvent relayé dans la littérature tend ainsi à délaisser tout un pan de l'approche zimmerienne de la composition cinématographique et s'avère peu adéquat pour analyser les partitions les plus récentes de Zimmer qui ne correspondent pas ou peu àceschème ${ }^{3}$.C'estlecas, en particulier, d'Interstellar, dontlabande-son excèdelargement

1 Sur la cinémusicologie, voir Huvet 2016.

2 Après la journée d'étude organisée que nous avons co-dirigée et co-organisée avec Jérémy Michot en novembre 2015 à l'Université Rennes 2, intitulée « Reconfiguration des pratiques musicales dans le cinéma hollywoodien contemporain ", un colloque international s'est tenu à l'Université de Rouen fin avril 2017 sous la direction de Cécile Carayol, Pierre Albert Castanet et Henning Lohner.

3 Voir notamment l'article d'Emmanuelle Bobée, "La partition sonore et musicale de Dunkerque (C. Nolan, 2017) : une expérience sensorielle inédite », dans le présent numéro. 
ce cadre général prédéfini et qui, à notre connaissance, n'a encore fait l'objet d'aucune analyse cinémusicologique détaillée ${ }^{4}$.

Le film raconte comment, alors que la Terre connaît une asphyxie de plus en plus préoccupante, une équipe d'astronautes est secrètement chargée d'explorer une nouvelle galaxie par-delà un trou de ver et de tenter de trouver une planète habitable parmi les trois où différents spationautes de la mission Lazare ont été envoyés quelques décennies plus tôt, pour fonder une colonie. Le personnage principal, Cooper, fait le choix déchirant d'abandonner son fils Tom et sa fille Murphy (surnommée Murph) pour tenter d'assurer le salut des siens et de l'humanité. Voyage intersidéral et tableau monumental, Interstellar se révèle ainsi avant tout dominé par « le deuil, 1'absence, la famille » (Berthomieu 2014, p. 41), focalisé sur l'amour entre un père et sa fille au-delà des dimensions de l'espace et du temps. Il s'agira dès lors de voir comment le traitement de la musique place l'humain au cœur du film, et de quelles manières Zimmer fait des liens entre Cooper et sa fille Murph le point de mire de sa partition. En filigrane, notre étude propose, à partir de l'exemple de ce film, une autre approche de l'esthétique du compositeur, qui se veut complémentaire au modèle dominant.

Nous établirons que l'esthétique de Zimmer apparaît fondamentalement souple et diversifiée, rehaussant subtilement les nœuds dramatiques où s'entrecroisent plusieurs fils narratifs. Nous verrons ensuite comment le compositeur dresse tout au long du film un portrait intime des relations humaines, avant de nous intéresser au paroxysme émotionnel et à la plénitude sonique d'Interstellar résultant des interactions entre musique et sound design et de la gestion du mixage.

\section{UNE ESTHÉTIQUE MUSICALE RICHE ET SOUPLE}

\section{Principales caractéristiques du style d'écriture de Hans Zimmer}

Hans Zimmer est présenté dans la littérature dominante comme le " maître d'œuvre » (Berthomieu 2004, p. 73) d'un nouveau style musical hollywoodien, désigné sous les appellations «style Zimmer » (De la Grange 2002, p. 89) ou " style Media Ventures ", d'après l'ancien nom de son studio. Comme le souligne Benjamin Wright, ses musiques possèdent un son distinctif tout d'abord en ce qu'elles s'appuient à la fois sur les pratiques compositionnelles de la période classique hollywoodienne, et sur une synergie entre l'orchestre et l'électronique (Wright 2015, p. 319). Elles se caractérisent en outre par une pulsation rythmique marquée, fréquemment générée par un ostinato aux cordes dans le registre médium, au-dessus duquel s'ajoutent des dessins rythmiques répétitifs et des accents aux percussions, ainsi qu'une basse au caractère dramatique. La mélodie est le plus souvent confiée aux cordes, sur lesquelles viennent se greffer les cuivres, en particulier les cors, doublés de synthétiseurs pour créer un son puissant. Sur le plan formel, «Zimmer bannit les longues mélodies au profit de motifs courts

4 Frank Lehman et Vasco Hexel ne font que pointer la singularité de la partition d'Interstellar, « l'écart stylistique clair» (" a clear stylistic departure», Hexel 2016, p. 7) et les «nouvelles directions » (" new directions», Lehman 2016, p. 51) explorées par Zimmer, sans donner davantage de précisions. 
et constamment répétés [...]. C'est l'augmentation du volume, la répartition variable de l'orchestre et des synthétiseurs qui servent de développement » (Berthomieu 2004, p. 74). Enfin, le langage harmonique employé par Zimmer est relativement simple ; il comporte beaucoup d'accords classés et de nombreux enchaînements fonctionnels ${ }^{5}$.

\section{Noeuds dramatiques et détournement des procédés d'écriture}

Ce modèle se heurte toutefois à certaines limites. Tout d'abord, certains des procédés identifiés par les chercheurs sont détournés dans Interstellar, utilisés dans une optique opposée à celle qui est la plus largement identifiée dans la littérature ; la partition s'éloigne alors d'une simple application de recettes musicales toutes faites et systématiques. Les procédés d'écriture observés visent ici à rehausser subtilement certains passages très tendus où se jouent des nœuds dramatiques essentiels.

La scène où Cooper lutte contre le Dr Mann et agonise sur la planète glacée tandis que, sur Terre, sa fille Murph décide de retourner dans sa maison d'enfance pour comprendre les indices laissés par son "fantôme ", est particulièrement saisissante à cet égard. Elle présente en effet la plupart des éléments caractéristiques du style d'écriture de Zimmer - tels la superposition progressive de différentes strates musicales, le recours aux ostinatos, la répétition inlassable d'un motif mélodique court non développé, et le bourdonnement produit par les infra-basses. Cependant, ces procédés d'écriture sont utilisés de manière relativement singulière, dans une réalisation beaucoup plus éthérée et obsédante. Le caractère épuré induit un traitement de l'orchestration, du rythme et du tempo diamétralement opposé à celui attendu chez Zimmer dans une scène d'action : on est loin de la « musique-excitation " (Berthomieu 2004, p. 73), des " percussions agressives » (Berthomieu 2013, p. 137) et de la " satur[ation] de cuivres hargneux » (Lauliac 1998, p. 103). Cette séquence d'Interstellar illustre au contraire la versatilité de l'esthétique zimmérienne et le faisceau de possibilités offertes par une même technique d'écriture. Le compositeur évoque d'ailleurs, au sujet de ses conversations avec Christopher Nolan, une volonté commune d'opter pour une approche renouvelée de la bande sonore du film par rapport à leurs précédentes collaborations :

Our sonic world became more and more defined and more specific during these discussions mainly by establishing rules about what we didn't want to do! No big epic action drums. Gone were the propulsive string ostinato that had worked so well for us over the past ten years, to be replaced by large sections of woodwinds, four concert grand pianos, harp and marimbas (Zimmer 2014, livret de la bande originale p. 4) ${ }^{6}$.

Le cue analysé, portant le titre "Coward ", commence au moment où Cooper vient de subir un premier assaut du Dr Mann, qui tente de le tuer pour pouvoir s'emparer de son vaisseau. Cooper comprend alors que Mann a falsifié toutes les données et que sa planète est en réalité stérile. Le cue débute de manière singulière

\footnotetext{
$5 \quad$ À ce sujet, voir Huvet 2015, p. 129.

6 À ce sujet, voir également Chitwood 2013, http://collider.com/interstellar-score-hans-zimmerdark-knight-trilogy/, consulté le 14 août 2017.
} 
par une ponctuation au timbre proche d'un marimba, comme le tic-tac d'une horloge qui décompte les minutes qu'il reste à vivre à Cooper ${ }^{7}$. La musique entretient une attente, mais la découverte de la traîtrise de Mann n'est pas soulignée musicalement. Un simple accord parfait de mi mineur est ensuite tenu aux bois en crescendo alors que les deux personnages s'empoignent violemment dans une lutte désespérée. Il produit un décalage par rapport à l'image par son caractère calme, suspendu, presque détaché : l'accompagnement musical ne transmet ici aucun sentiment d'urgence, et ne tente pas d'illustrer l'affrontement âpre entre Cooper et le Dr Mann par un synchronisme ou par du mickey-mousing. Ce sentiment de détachement est accentué par le zoom arrière qui cadre les personnages en plan général, les transformant en deux taches blanches perdues dans l'immensité glaciaire et accentuant ainsi leur solitude extrême, leur petitesse au sein de l'univers. Le scénario indique ainsi : "Wider [shot] shows us two tiny figures in a vast landscape, deciding the future of humanity with a brawl..." (frères Nolan 2014, p. 106).

La ponctuation de marimbas se poursuit sur les plans suivants du montage alterné, soulignant l'impuissance de Murph à convaincre son frère de quitter la maison pour sauver sa famille (extrait vidéo 1).

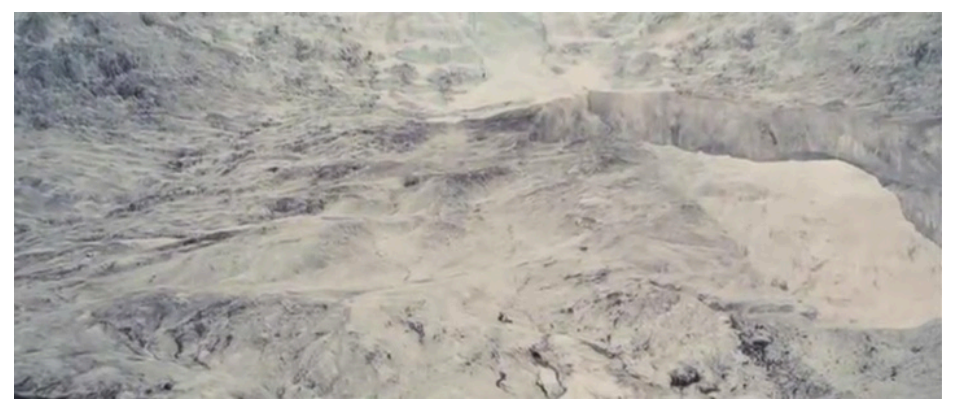

Extrait vidéo 1 : Christopher Nolan, Interstellar, Mann attaque Cooper après avoir avoué sa lâcheté, 01:53:01-01:54:07 (C) Warner.

La suite de la scène témoigne d'un travail de détail méticuleux en matière d'orchestration : au lieu de se fondre, le timbre de chaque instrument reste clair et distinct. En outre, le choix même des instruments s'avère relativement singulier : loin de recourir à une formation, plus habituelle chez lui, de cordes ou de cuivres et percussions, Zimmer opte pour un ensemble de bois fourni, composé de 6 pupitres de flûtes, 4 pupitres de hautbois, 6 pupitres de clarinettes et 4 pupitres de bassons (selon le livret de la bande originale), et surtout un orgue et un piano solistes, tandis que les cordes et les percussions sont placées en retrait. En dépit de cet ensemble de bois imposant, le rendu sonore n'est pas celui d'une masse écrasante et d'une musique-

$7 \quad$ Le tic-tac de l'horloge revient tout au long du film sous différentes formes. Parfois très proche d'un bruitage pur, il est entremêlé à la musique, notamment dans la séquence où Cooper, Doyle et Amelia amerrissent sur la planète océanique, où le temps est beaucoup plus étiré que sur Terre (une heure sur la planète de Miller équivaut à sept années terrestres), et lorsque Cooper prend la décision de s'amarrer à l'Endurance après l'explosion provoquée accidentellement par le Dr Mann. Symbolisant le temps précieux qui s'écoule, ce son récurrent est étroitement lié au désir de Cooper de retrouver sa famille, et en particulier sa fille Murph. 
excitation saturée ; la sensation est plutôt celle d'un son plein et " rond " mettant en valeur les timbres épurés. Alors que Cooper agonise, en manque d'oxygène, et que Mann se détourne, Zimmer superpose trois strates (exemple 1), dont une dans l'extrême grave, et deux aux sonorités cristallines.
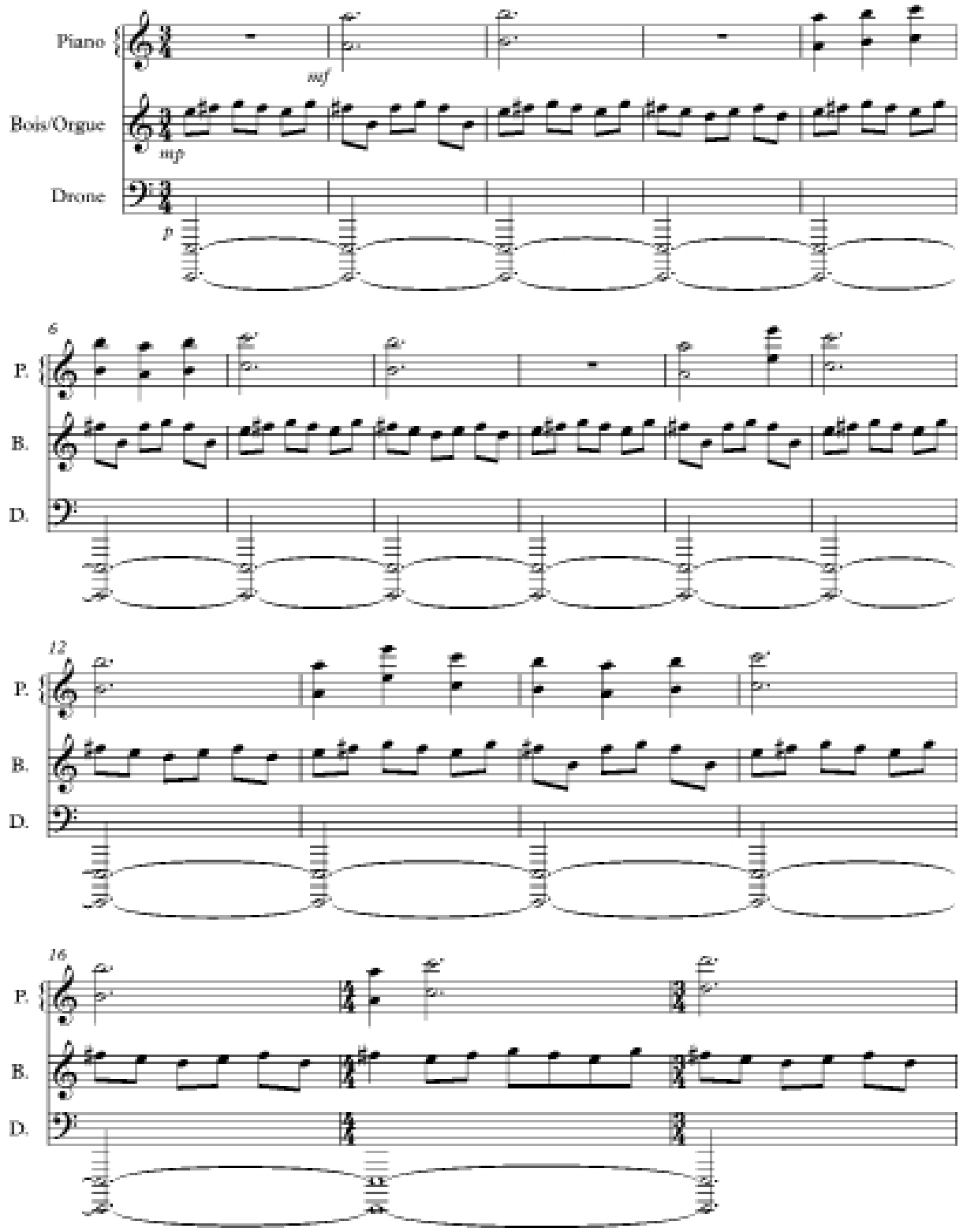

Exemple 1: Hans Zimmer, Interstellar, cue "Coward», superposition de trois strates.

Transcription personnelle à partir de la bande originale (04:00-04:55).

Sur un drone tenu aux pédalier et synthétiseur se développe un ostinato de croches circulaire aux bois et à l'orgue dans l'aigu, dans le mode de la sur mi, dont le profil serpentin revêt une dimension obsédante. Il est lui-même surmonté d'une mélodie très délicate, au caractère doux et mélancolique, énoncée au piano dans l'aigu et construite sur la, qui semble suspendre le temps - impression de suspension renforcée par la forte réverbération affectant le timbre du piano. La mélodie renforce le caractère poignant 
de l'aveu du Dr Mann, incapable de supporter la vue des souffrances de l'astronaute et qui, malgré son geste meurtrier, tient à accompagner ses derniers instants par sa voix. Mais la musique crée également une distance par rapport à l'agonie terrible de Cooper qui, elle, est mise en valeur d'une part, visuellement, par les mouvements saccadés de la caméra qui suit les moindres tressautements du personnage en plan rapproché et, d'autre part, sur le plan sonore, par les grognements et les halètements désespérés de Cooper, mixés au premier plan et entendus de très près de manière à renforcer l'empathie et l'identification du spectateur avec le personnage.

Une amplification dramatique est ensuite créée par un changement dans la primauté des lignes et le choix d'orchestration : l'ostinato passe au premier plan, dans une nuance $f$, et devient la mélodie principale (exemple 2), tandis que le relais de timbres, des bois à l'orgue, lui confère une dimension particulièrement majestueuse tout en accentuant son caractère hypnotique par ses sonorités épurées. Cette amplification sonore est là encore frappante en ce qu'elle ne crée pas de sensation de masse imposante, mais bien plutôt un effet de plénitude sonore.
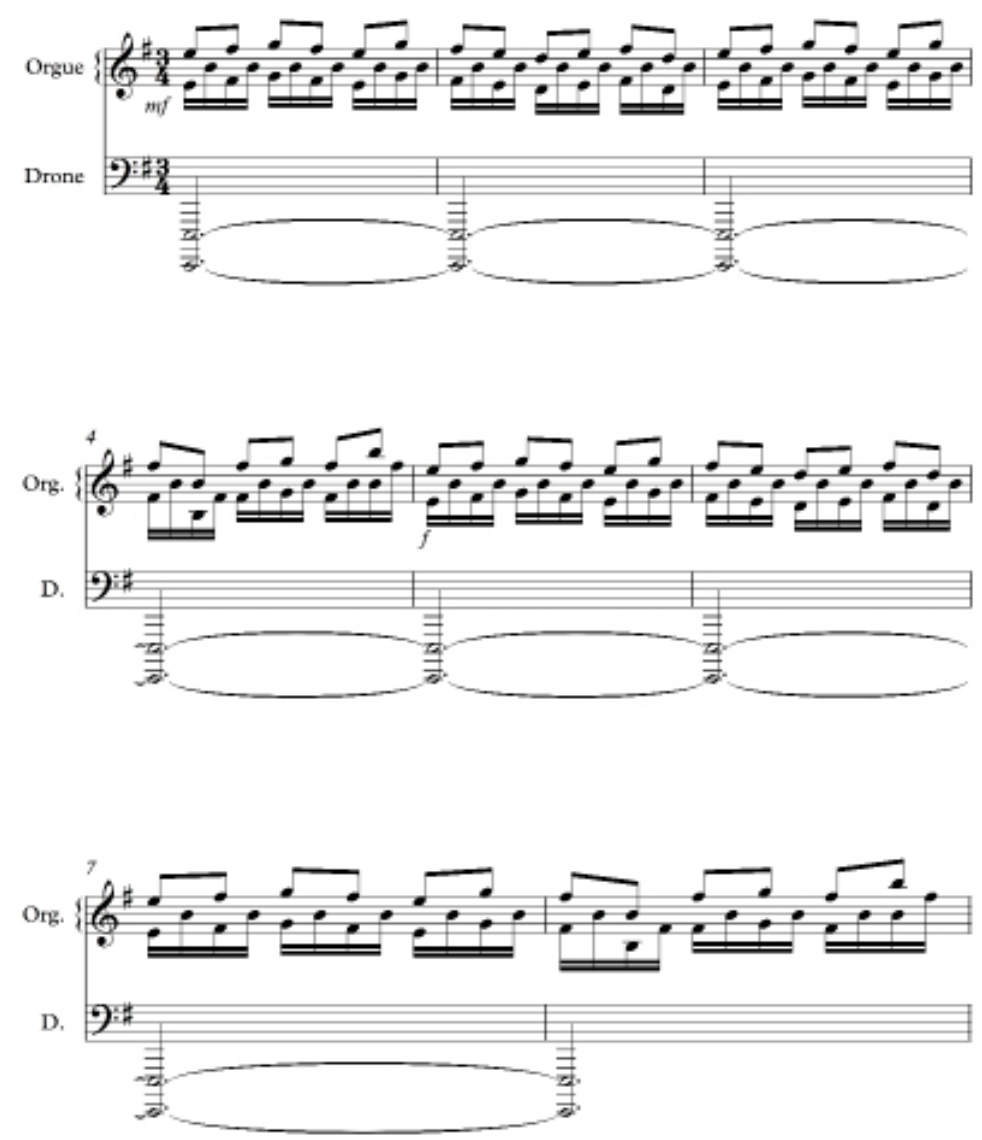

Exemple 2: Hans Zimmer, Interstellar, cue "Coward», l'ostinato devient la mélodie principale. Transcription personnelle à partir de la bande originale (04:56-05:19).

L'effet de transe suscité par l'emprunt aux techniques d'écriture de la musique minimaliste, entretient par ailleurs un rapport singulier et étroit avec le dialogue : 
la partition se marie parfaitement à la voix de l'acteur Matt Damon (interprétant le Dr Mann) qui, alors qu'il récite le début du poème de Dylan Thomas « Do not go gentle into that good night » $(1947)^{8}$, se distingue par un débit vocal particulier, à la manière d'une litanie. Ce poème revêt en lui-même une dimension incantatoire par la répétition "Rage, rage against the dying of the light », employée comme refrain un tercet sur deux selon la forme traditionnelle des villanelles :

Do not go gentle into that good night, Old age should burn and rave at close of day; Rage, rage against the dying of the light.

Though wise men at their end know dark is right, Because their words had forked no lightning they Do not go gentle into that good night.

Good men, the last wave by, crying how bright

Their frail deeds might have danced in a green bay, Rage, rage against the dying of the light.

À cette répétition du vers "Rage, rage against the dying of the light " dans le film font écho, de manière remarquable, les boucles mélodico-rythmiques minimalistes de l'accompagnement musical. Alors qu'elle se poursuit sur le montage alterné, la voix off exprime une forme de détachement par rapport à l'angoisse et à la détresse extrêmes de Cooper, tout comme Mann ferme les yeux et coupe son transmetteur pour ne plus entendre ses halètements, comme s'il s'adressait avant tout à lui-même en récitant ces vers.

Malgré le caractère de plus en plus critique de la situation, il est important de noter que la musique se poursuit de manière quasi identique, dans un tempo relativement lent par rapport à d'autres séquences tendues similaires de la saga Pirates des Caraỉbes, de Gladiator (Ridley Scott, 2000), ou du film Anges \& Démons (Ron Howard, 2009). L'accompagnement musical ne présente pas de dramatisation au sens d'une accentuation ou d'une emphase particulière sur telle action ou telle réplique. Il ne connaît ainsi aucune modification notable lorsqu'Amelia, prévenue in extremis, vole au secours de Cooper : la musique ne suit pas le déroulement de la séquence, mais semble plutôt se déployer à la manière d'un discours parallèle à celle-ci.

Alors que la scène s'avère très dramatique, portant la tension et le suspense à un point d'acmé, l'approche choisie ici par Zimmer fait donc valoir plusieurs décalages subtils entre la musique et l'image, sur le plan du caractère et de la temporalité. $\mathrm{Au}$ lieu d'illustrer l'affrontement physique entre les deux hommes ou d'appuyer l'agonie de Cooper, la musique dessine une mélopée répétée inlassablement, dont le caractère obsédant, surplombant et relativement détaché par rapport à l'action, est accentué par le traitement des lignes mélodiques et les choix de timbres instrumentaux.

8 Après une première publication en 1951 dans le périodique Botteghe Obscure, le poème de Dylan Thomas est ensuite inclus dans le recueil In Country Sleep, And Other Poems (1952). 
Elle invite alors à nuancer l'affirmation selon laquelle chez Zimmer, la « composition refuse toute complexité, toute orchestration singulière, et se destine à souligner la figuration physique d'une action »(Berthomieu 2004, p. 73). L'accompagnement orchestral crée un effet de suspens hypnotique qui surligne subtilement la dimension tragique de la scène par son caractère mélancolique, sans pour autant verser dans un soulignement emphatique ou un quelconque excès mélodramatique. Se déroulant quasiment sans modification tout au long de la scène, l'accompagnement unifie les actions concomitantes présentées en montage alterné et lie ainsi étroitement l'agonie de Cooper et le refus de Murph de renoncer à sauver la famille de son frère, comme si l'issue heureuse de l'une dépendait de la prise de décision de l'autre : Murph et Cooper mènent chacun de leur côté une lutte capitale, l'un pour sa survie, l'autre pour celle de ses proches. Enfin, la même musique s'appliquant à tous les personnages sans variation en fonction de la présence de l'un ou de l'autre à l'image, Mann est dépeint moins comme un meurtrier impitoyable que comme une figure tragique, un astronaute héroïque déchu, dont les failles résident justement dans son incapacité à accepter de vivre coupé de tout lien avec le reste de l'humanité jusqu'à la fin de ses jours et de mourir dans la solitude la plus absolue (extrait vidéo 2).

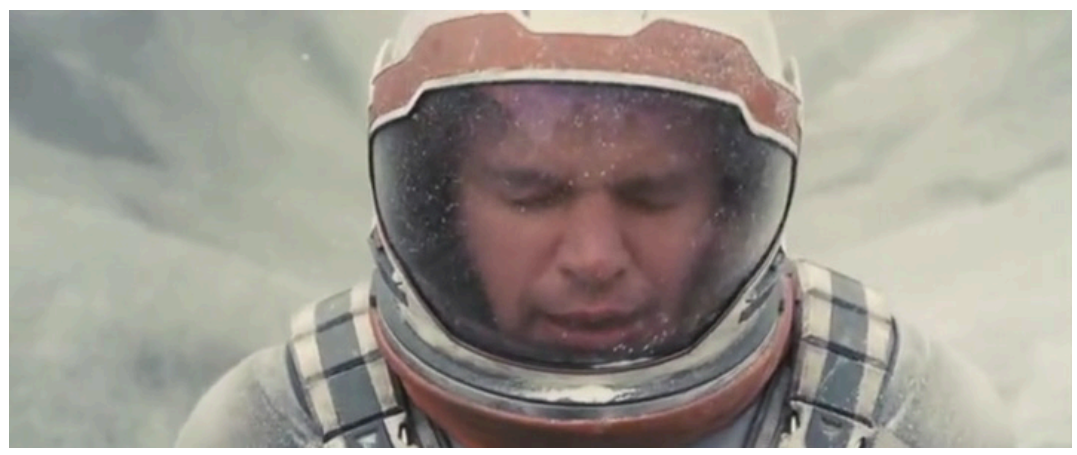

Extrait vidéo 2: Christopher Nolan, Interstellar, Cooper agonise sur la planète glacée tandis que, sur Terre, Murphy fait demi-tour pour retourner vers la maison de son frère, 01:55:54-01:58:01 @ Warner.

\section{UN PORTRAIT INTIME DES RELATIONS HUMAINES}

Outre le fait que le modèle stylistique dégagé dans la littérature dominante n'est donc pas toujours adéquat pour analyser les partitions de Zimmer, certains procédés d'écriture déployés par le compositeur tendent par ailleurs à être occultés. Or, ils contribuent pleinement dans Interstellar à dresser musicalement un portrait intime des relations humaines et à rehausser la réflexion proposée autour des liens humains et familiaux, et de l'absence des êtres chers. Nous nous intéresserons ici au traitement minutieux de l'orchestration, à l'absence de pulsation rythmique marquée, et à l'écriture mélodique du «thème d'amour » de Cooper et Murph. 


\section{Un travail sur les timbres transparents}

Alors que les publications existantes insistent majoritairement sur la production d'un son puissant et massif dans les partitions de Zimmer, le travail du compositeur sur les timbres transparents et les sonorités épurées est fréquemment laissé de côté, alors que Zimmer emploie assez fréquemment ce genre de sonorités ${ }^{9}$. De nombreux cues d'Interstellar privilégient les orchestrations légères, tel "Afraid of Time ", qui débute juste après que Murph ait laissé un message à son père en le suppliant de rentrer. Le cue est caractérisé par la chatoyance et la clarté des timbres. Il s'ouvre par un mi tenu dans le suraigu au synthétiseur, qui nimbe les autres interventions instrumentales d'un halo lumineux (exemple 3). Quelques notes perlées, un intervalle de seconde mineure alternativement descendante et ascendante, s'échangent en relais entre la harpe et le piano, affecté d'une légère réverbération à la manière d'un halo.

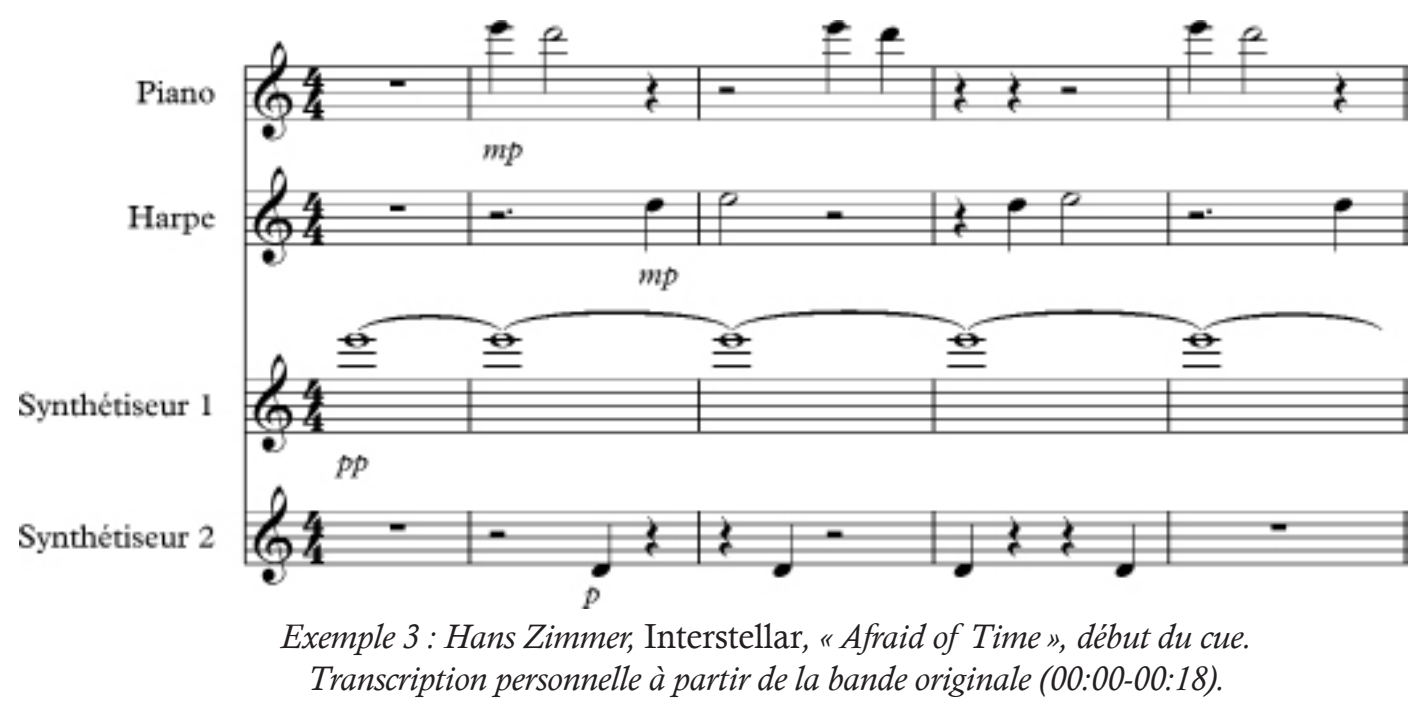

Un ostinato très doux débute ensuite au synthétiseur, construit sur une quinte à vide ré-la, qui ajoute à l'effet de transparence (exemple 4). Enfin, le piano égrène de façon brisée des accords parfaits parallèles sur ré, agrémentés de septièmes et de neuvièmes expressives, auxquels le registre extrême aigu confère une couleur claire et cristalline.

9 Nous renvoyons à ce sujet à l'article de Cécile Carayol, «La Ligne rouge de Hans Zimmer : matrice d'un "nouvel Hollywood" électro-minimaliste et contemplatif » dans le présent numéro. 

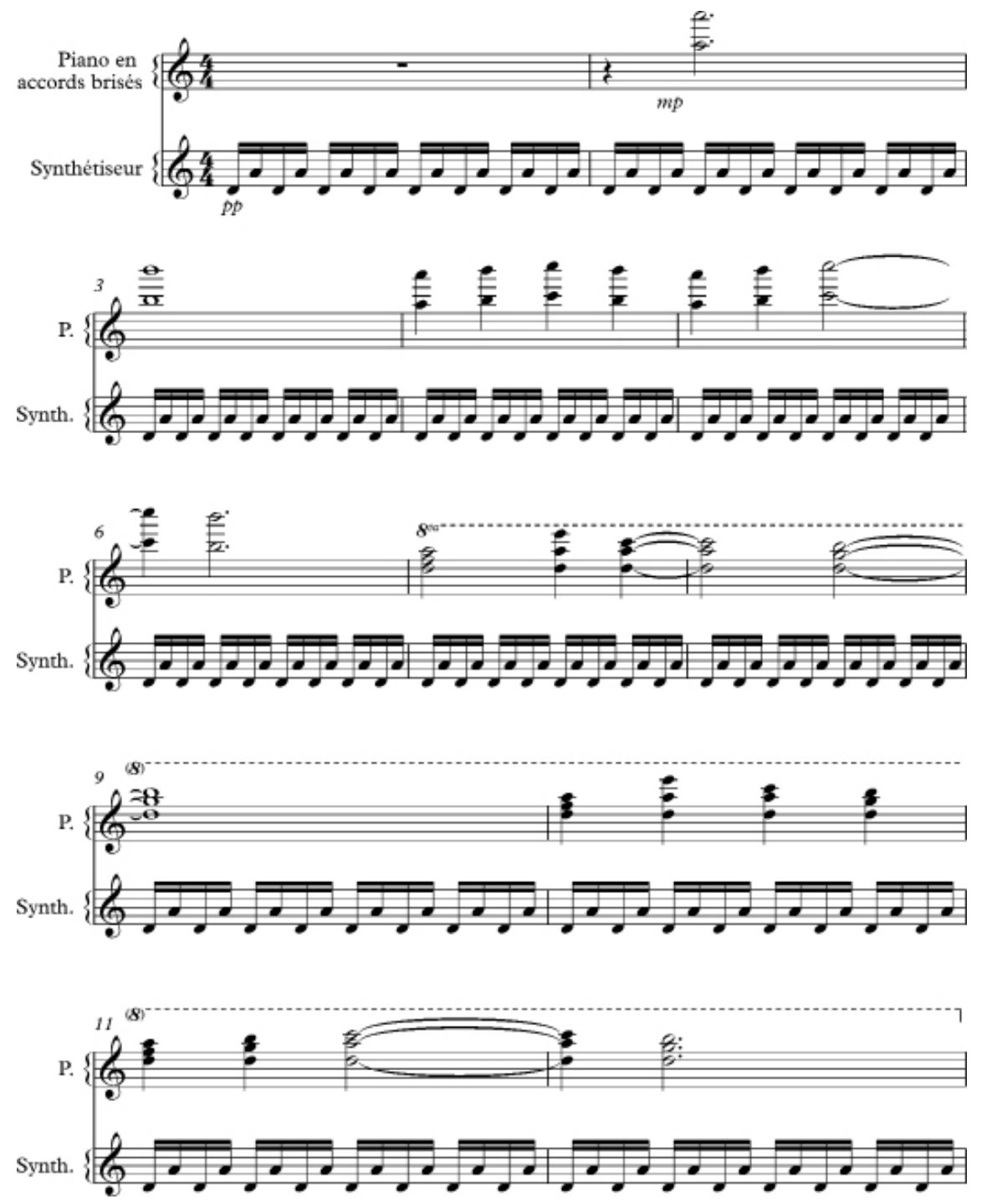

Exemple 4 : Hans Zimmer, Interstellar, "Afraid of Time ", suite du cue.

Transcription personnelle à partir de la bande originale (00:19-01:08).

L'écriture rappelle le "style tintinnabuli » ${ }^{10}$ développé par le compositeur estonien Arvo Pärt, dont il retrouve le tempo lent, la recherche de résonance, et les sonorités carillonnantes. Zimmer en livre cependant une interprétation personnelle par un caractère plus affligé et mélancolique que contemplatif, l'absence de toute connotation mystique et la présence d'un ostinato en croches en arrière-plan, qui rompt avec le temps lisse recherché par Pärt. Ce travail sur les timbres transparents et les sonorités épurées ainsi que la mélancolie portée par la ligne mélodique soulignent tout en

10 Jacques Viret le définit ainsi : «Il s'agit d'une technique d'écriture inspirée par le chant grégorien et les polyphonies primitives et fondée sur un contrepoint à deux voix, l'une d'entre elles se déplaçant mélodiquement - par mouvements le plus souvent conjoints - dans un mode diatonique, tandis que l'autre fait entendre les notes principales (triade, accord parfait) de ce même mode » (Viret 2001, p. 362). 
délicatesse le chagrin de la jeune femme puis la souffrance liée à l'absence des êtres aimés lors du dialogue entre Murph et le professeur Brand, alors que celui-ci évoque Amelia et lui fait part de ses angoisses face au temps qui passe (extrait vidéo 3 ).

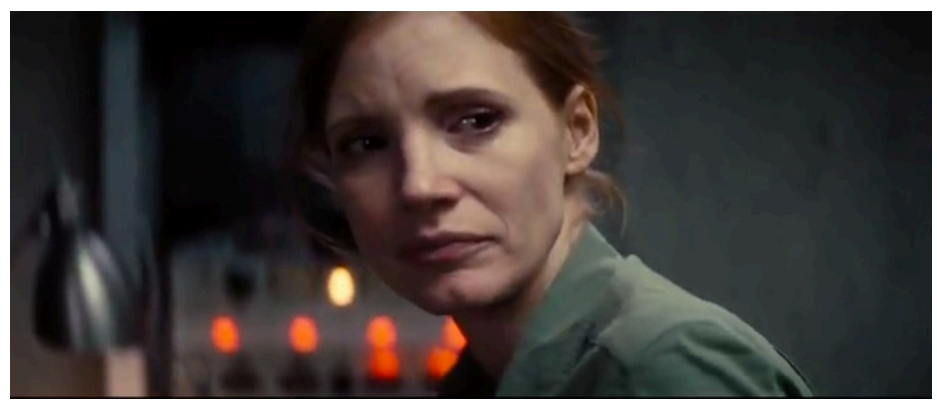

Extrait vidéo 3 : Christopher Nolan, Interstellar, le professeur Brand Murph évoque auprès de Murph l'absence des êtres chers et sa crainte du temps qui passe, 01:22:48-01:24:21 @ Warner.

Une temporalité suspendue

Un autre élément important mérite d'être souligné : bien que la pulsation soit un élément phare de son esthétique, Zimmer travaille pourtant le plus souvent à son effacement dans Interstellar ${ }^{11}$, dont la plupart des cues sont caractérisés par une absence d'accents marqués. Dans la scène où Cooper part avec le Dr Mann sécuriser trois sites sur la planète glaciaire avant de repartir sur Terre et que Murph rend visite à la famille de son frère dans leur maison d'enfance, par exemple, une temporalité très étirée est créée par les longues tenues de cordes, au caractère aérien et planant, auréolées d'un halo nébuleux de synthétiseur. Dans les sections relatives à Cooper, ce dépouillement et cette épuration musicale poussée à l'extrême entrent ici en résonance avec le "minimalisme chromatique " de l'image, les " entités plastiques raréfiées " (Berthomieu 2014, p. 40) et la " symétrie des reliefs minéraux » de la planète de Mann (ibid., p. 41), figée dans une glace grisâtre inhospitalière ; tandis que dans les plans sur Terre, l'accompagnement musical crée un effet de suspens, enveloppant la remémoration triste de Murph du départ de son père d'un voile doux et triste, en écho aux paroles de Mann insistant sur l'importance primordiale des liens humains. La musique met ainsi le dialogue en valeur et semble figer l'instant, précédant immédiatement le tournant dramatique et l'enchaînement tragique des événements quelques instants plus tard avec, d'une part, l'éclatement d'une violente dispute entre Murph et son frère Tom et, d'autre part, la tentative d'assassinat de Cooper par le Dr Mann (extrait vidéo 4).

11 Cet aspect se retrouve également dans d'autres films, comme le montre notamment Cécile Carayol dans son article du présent numéro, "La Ligne rouge de Hans Zimmer : matrice d'un "nouvel Hollywood" électro-minimaliste et contemplatif ». 


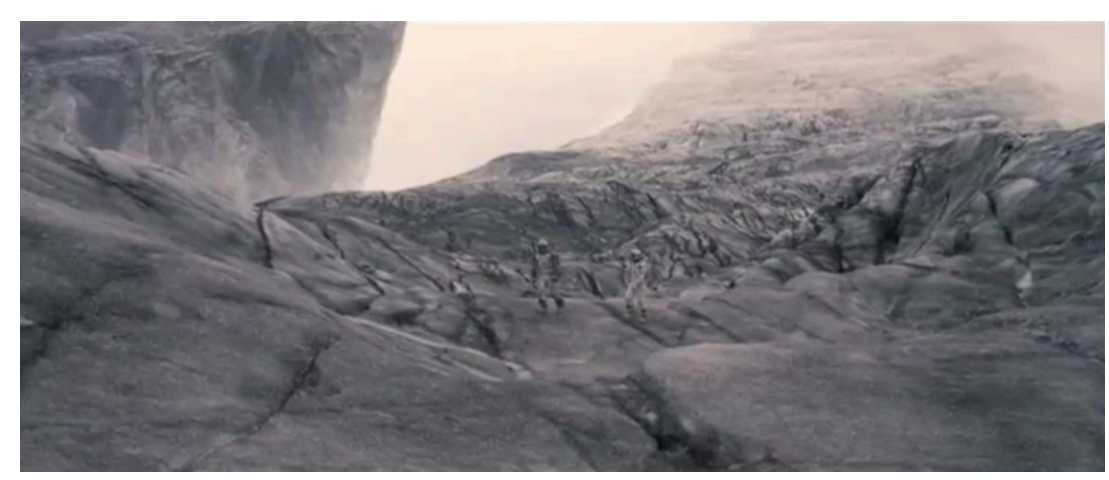

Extrait vidéo 4: Christopher Nolan, Interstellar, Cooper part avec le Dr Mann sécuriser trois sites sur la planète glaciaire tandis que Murph rend visite à la famille de son frère, 01:48:43- 01:49:43@ Warner.

\section{Le thème d'amour de Cooper et Murph}

Si Interstellar propose un voyage spectaculaire vers l'inconnu et les espaces infinis au-delà de notre système solaire, cette fresque « visant au monumental » (ibid., p. 41) ne sacrifie pas pour autant l'humain. Au contraire, c'est véritablement l'amour père-fille qui est au cœur du film. La relation entre Cooper et Murph est mise en valeur par le thème principal écrit par Zimmer, qui est de l'aveu du compositeur lui-même un thème d'amour entre le père et sa fille, exprimant une idée de "solitude mélancolique » (Zimmer 2014, livret de la bande originale p. 4) ${ }^{12}$. À la différence des grands thèmes soumis à des développements dans les partitions des symphonistes de l'âge d'or hollywoodien ou de John Williams, la simplicité du thème, basé sur quelques notes, est revendiquée par le compositeur :

I write really simple music. [...] There isn't a theme I've written that you can't play with one finger on the piano. [...] [W] 're all capable of amazing everybody else with complications. But I think there's something really nice if things are just minimalistic and transparent in a way (Zimmer, cité dans Grieving 2013).

Outre l'extrême dépouillement du matériau musical, le thème est surtout caractérisé par la couleur particulière que lui confère l'orgue. Il s'agit de l'instrument phare d'Interstellar, jamais utilisé auparavant par Zimmer comme soliste, dans une captation directe effectuée sur l'orgue de Temple Church à Londres, restauré en 2013. Dans le thème d'amour écrit pour Cooper et Murph, son utilisation est très différente de celle que peut en faire Danny Elfman par exemple, chez qui l'orgue sert souvent l'évocation spectaculaire d'un univers surnaturel, féérique ou fantastique (comme dans Batman (1989), Charlie et la chocolaterie (2005) ou Frankenweenie (2012)). De son côté, Zimmer l'utilise ici majoritairement dans le registre médium-aigu pour ses sonorités épurées, dans un caractère délicat et suspendu, presque fragile - bien qu'il l'emploie dans une optique véritablement grandiose dans d'autres passages du film, comme on le 
verra plus loin. Dans la scène où Cooper accède à un espace-temps tridimensionnel et envoie à sa fille un message codé en morse pour qu'elle l'empêche de partir pour l'espace, le thème, lent, recueilli et nostalgique, est accompagné par un cluster tenu en valeurs longues au synthétiseur (exemple 5).
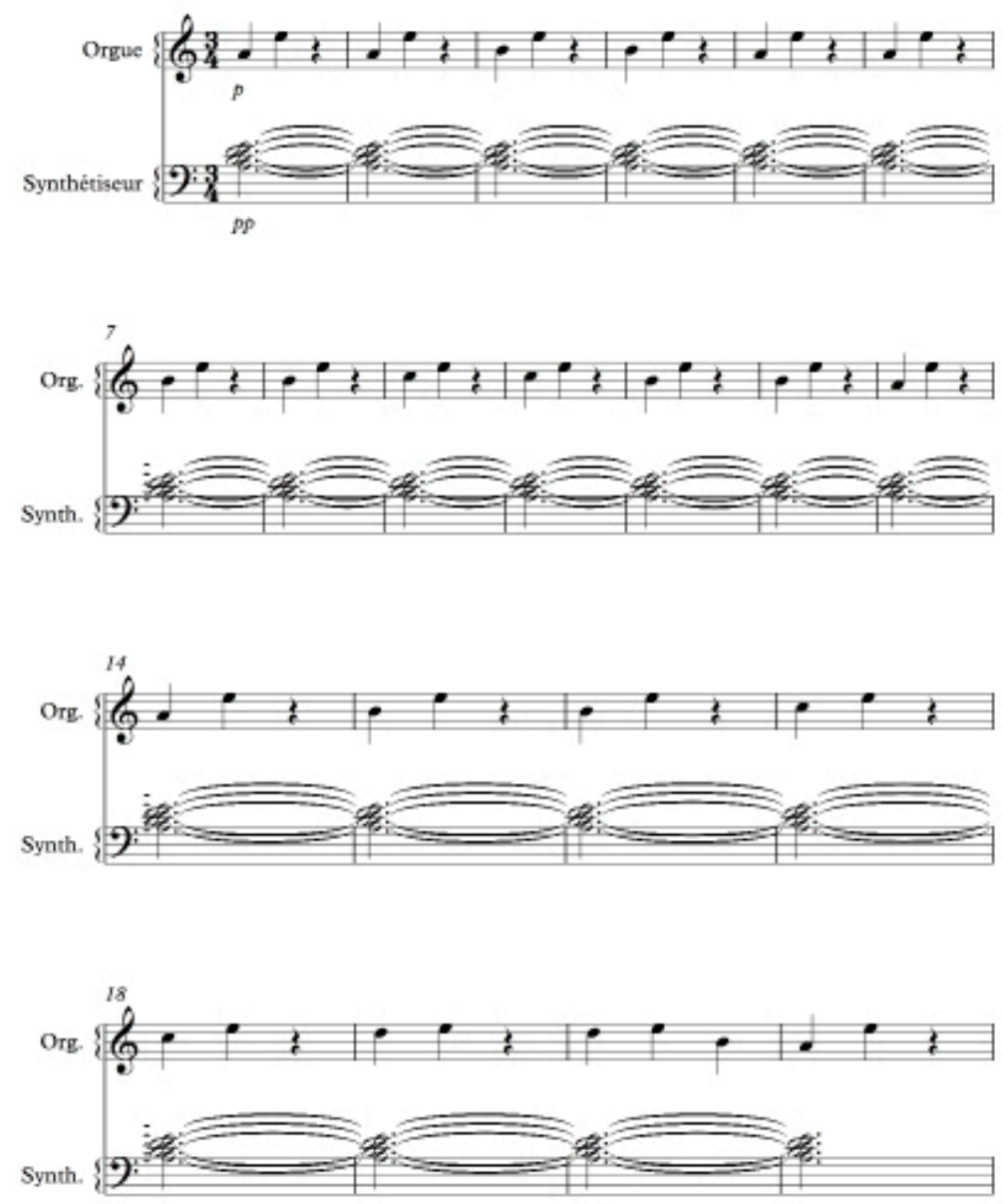

Exemple 5 : Hans Zimmer, Interstellar, cue "S.T.A.Y. », thème d'amour de Cooper et Murph. Transcription personnelle à partir de la bande originale (00:42-01:06).

Il est varié peu à peu dans ses intervalles, par l'ajout de notes de passage, puis par l'entrée des basses jouées au pédalier en même temps que le gros plan sur le message "STAY» décrypté par la fillette. Ces variations éloignent le thème du modèle principal dégagé dans la littérature - une écriture basée exclusivement sur des motifs très brefs répétés à l'identique, et dénuée de grand thème véritable : la raréfaction du matériau musical n'exclut pas pour autant le déploiement progressif du thème d'amour.

Là encore, comme dans la scène de l'agonie de Cooper analysée précédemment, Zimmer opte pour un certain détachement par rapport à l'image et évite le piège du sentimentalisme larmoyant alors que se joue ici un véritable climax dramatique :

Dans l'espace replié sur lui-même du wormhole, deux instants lointains coïncident. Aux confins de la galaxie, l'espace intime de la maison du héros est dupliqué en relief $[\ldots]$, cyberespace du temps qui balaie la rêverie enfantine sur l'espace et les fantômes. Tout se trouve dans la bibliothèque, premier plan du film comme résolution finale (Berthomieu 2014, p. 41). 
La musique n'appuie pas la tragédie familiale qui se noue ici, ni les cris de désespoir et la rage de Cooper, ni la compréhension soudaine de Murph que son "fantôme " n'était autre que son père lui-même et sa joie émue : la partition nimbe l'ensemble de la scène d'un halo mélancolique très doux sans en suivre l'évolution formelle et dramatique (extrait vidéo 5).

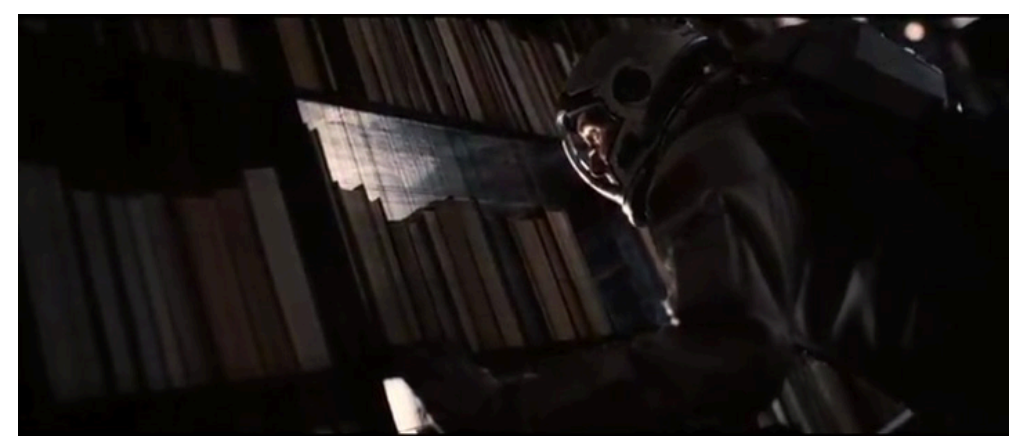

Extrait vidéo 5: Christopher Nolan, Interstellar, Cooper observe Murph depuis le trou de ver alors que celle-ci découvre l'identité de son "fantôme", 02:24:48-02:26:45 (c) Warner.

\section{PAROXYSME ÉMOTIONNEL ET PLÉNITUDE SONIQUE}

Hypnose optique et sons extatiques

Tout en optant pour une retenue généralisée dans l'ensemble du film, Zimmer ponctue également certains plans ou scènes de cette fresque monumentale par des effets paroxystiques lorsque sa musique s'entremêle aux effets sonores, voire joue le rôle d'un bruitage. Ces effets de paroxysme sonore confrontent la petitesse de l'homme et la force de ses émotions à l'infiniment grand et à la majesté glacée de l'espace.

Un pan essentiel de l'esthétique zimmérienne, assez peu évoqué dans la littérature dominante, réside en effet dans une conception globale et intégrative de la bande sonore $^{13}$, suscitant de nombreuses interactions saisissantes entre la musique et les effets sonores. Sur Interstellar, le compositeur nourrit ses expérimentations de l'inspiration que provoque chez lui le mécanisme des tuyaux de l'orgue. Élaborant des sons à partir des imaginaires aérien et éolien, il utilise les chœurs à contre-emploi, s'intéressant avant tout à la corporéité du souffle et travaillant le matériau musical comme une pure matière sonore :

Istarted synthesizing sounds of air and wind. Large choirs became an investigation of exhales rather than melodies, and creating impossible to sing soft harmonies which served as a human reverb for the pianos (Zimmer 2014, livret de la bande originale p. 4. Soulignement par le compositeur).

13 Voir en particulier Wright 2015, et les articles de Jérôme Rossi («Essai de caractérisation de l'évolution des musiques super-héroïques de Batman (1989) à The Dark Knight Rises (2012) ») et d'Emmanuelle Bobée ( La partition sonore et musicale de Dunkerque (C. Nolan, 2017) : une expérience sensorielle inédite ») dans le présent numéro. 
La porosité des frontières entre musique et sound design n'est bien sûr pas limitée aux partitions de Zimmer ; elle emblématise une approche de plus en plus répandue dans les blockbusters contemporains, corrélée en partie aux technologies numériques et à l'accroissement global de la palette sonore qu'elles permettent ${ }^{14}$. La particularité du compositeur allemand réside toutefois dans l'utilisation extensive qu'il en fait dans chacune de ses partitions et sa recherche constante de fusion entre timbres orchestraux et électroniques. Ayant à cœur d'entretenir une collaboration étroite avec les sound designers, Zimmer ne conçoit pas la composition et le travail sonore qu'il effectue sur ses synthétiseurs comme étant deux tâches séparées et exclusives l'une de l'autre :

I would have been just as happy being a recording engineer as a composer. Sometimes it's very difficult to stop me from mangling sounds, engineering [...], and actually getting me to sit down and write the notes. [...] There's a lot of things to be gotten out of making the sound designers into part of your band. We're all filling the same canvas (Zimmer, cite dans Grieving 2013).

En outre, cette manière d'envisager le son dans sa globalité est à la source même de la singularité de l'approche thématique du compositeur où, souvent, c'est le timbre, la couleur générée par l'harmonie, le registre, le rythme, le mode de jeu et la dynamique - donc la matière sonore même - qui sert de thématique. Zimmer affirme ainsi : "The colors are as important to me as the notes" (ibid.). Cette affirmation est étayée par l'analyse que fait Benjamin Wright du thème de Joker dans The Dark Knight: selon lui la matière sonore revêt le rôle de leitmotiv du personnage, caractérisé par une note de violoncelle

jouée lentement et avec rudesse, pour produire un son âpre, proche d'un rasoir. [...] La musique de Danny Elfman pour la scène d'ouverture du Batman de Tim Burton (1989), introduit une succession de thèmes wagnériens à la texture orchestrale dense, tandis que celle de Zimmer pour The Dark Knight de Christopher Nolan opte pour un micro-univers sonore présentant la note tenue du Joker. Dans le cas de Zimmer, c'est le timbre du violoncelle, et non sa mélodie, qui en est l'identifiant caractéristique (Wright 2015, p. 323) ${ }^{15}$.

Un travail similaire sur la matière sonore caractérise Interstellar, dont la bande sonore s'affirme comme un lieu d'expérimentation et de contrastes extrêmes : dans l'enchaînement des séquences et parfois même au sein d'une même scène, le silence complet de l'espace succède à un mur sonore épais où musique et effets sonores sont mixés à un volume extrêmement fort. Ce parti pris esthétique n'a d'ailleurs pas été sans susciter une polémique (comme à chaque sortie de film de Nolan), le réalisateur, le sound designer et le compositeur se voyant accusés d'avoir généré une bande-son

14 À ce sujet, voir notamment Huvet, à paraître.

15 "played slowly and roughly to produce a ragged, razor-like sound. [...] Danny Elfman's opening title music for Tim Burton's Batman (1989) introduces a suite of densely orchestrated, Wagnerian themes, while Zimmer's main title for Christopher Nolan's The Dark Knight opts for a micro sound world featuring the Joker's cello sustain. In Zimmer's case, it is the timbre of the cello, not its melody, which carries its identifying features ". 
médiocre, brouillée et saturée, le plus souvent au détriment du réalisme et de la clarté du dialogue. Or, comme le pointe justement Katie Kilkenny, " Nolan n'aspire pas au réalisme, il désire frapper les esprits ${ }^{16}$ (Kilkenny 2014). En outre, ce choix esthétique fait sens par rapport à la narration, comme le rappelle Kilkenny :

In the context of a movie that embraces the idea that emotions and feeling are the most important dimension of human experience, the overwhelming nature of the score is all part of the way Interstellar works as a cohesive whole (ibid.).

Dans le film, ce paroxysme sonique où la musique se fait matière sonore intervient dans deux cas de figure. Il traduit d'abord la petitesse de l'homme face au mystère et à l'immensité de l'univers, confrontant à l' « hypnose optique » des images cosmiques une forme d'équivalent sonore extatique ; ainsi, se crée dans Interstellar un "équilibre entre sidération intellectuelle et sensation physique "(Berthomieu 2014, p. 41). Mais il emblématise aussi la force des liens familiaux et l'amour reliant Cooper et Murph, comme on le verra plus loin. La puissance sonore recherchée a bien sûr à voir avec le paradigme d'une immersion spectatorielle « intensifiée » à l'ère numérique, les technologies permettant une mise en vibration du corps et une implication physique à un degré inédit, notamment grâce à une palette de fréquences et un éventail de dynamiques "impossibles auparavant"(Cathé 2007, p. 61) ${ }^{17}$. Ces possibilités techniques accroissent l'impact viscéral des bruitages aussi bien que des sons orchestraux, autant ressentis qu'entendus grâce à une puissance sonore exerçant une pression jusqu'à dix fois supérieure au son analogique (Sobchack 2005, p. 15), qui s'accorde particulièrement avec les effets visuels numériques spectaculaires innervant les blockbusters contemporains. Cette optique de sidération immersive a d'ailleurs participé au choix de l'orgue comme instrument majeur d'Interstellar comme le relate le compositeur, dont les choix lexicaux renvoient à la notion d'impact physiologique :

The enormous power of air within [the organ's] bellows pushing through thousands of huge pipes creates a sound sometimes so low and powerful it's like a fist punching into your solar plexus [...]. The acoustics created around this organ by the cavernous environment of Salisbury Cathedral amplifies the vast soundscape such that it becomes totally immersive (Zimmer 2014, livret de la bande originale p. 4).

Dans la scène où les astronautes quittent la Terre (extrait vidéo 6), après un long crescendo, l'accord qui retentit à pleine puissance à l'orgue s'apparente ainsi à un pur matériau sonore, envahissant et enveloppant totalement le plan général du vaisseau qui s'éloigne lentement de la planète vue de l'espace en tournoyant gracieusement sur lui-même. Emblématisant le désir de Zimmer de "générer une expérience viscérale pour les spectateurs qui seraient cloués à leurs sièges " ${ }^{18}$ (Zimmer, cité dans Lowder 2014), cet accord fff crée une sorte de "mur » sonore et suscite une forme

16 "Nolan doesn't aspire to realism; he aspires to be remembered."

17 Voir à ce sujet Huvet à paraître et Wood 2013, p. 420.

18 "I wanted [the viewers] to go and have the visceral experience of being pinned in their seats. " 
de plénitude sonique immersive d'autant plus saisissante qu'un grand silence lui fait suite sur un écran noir pendant plusieurs secondes. Selon un phénomène relevant de la psychoacoustique, un son parait en effet comparativement plus fort lorsqu'il est précédé ou suivi d'un silence complet (Kerins p. 61) ; Zimmer crée une sorte d'équivalent sonore au sublime monumental des images.

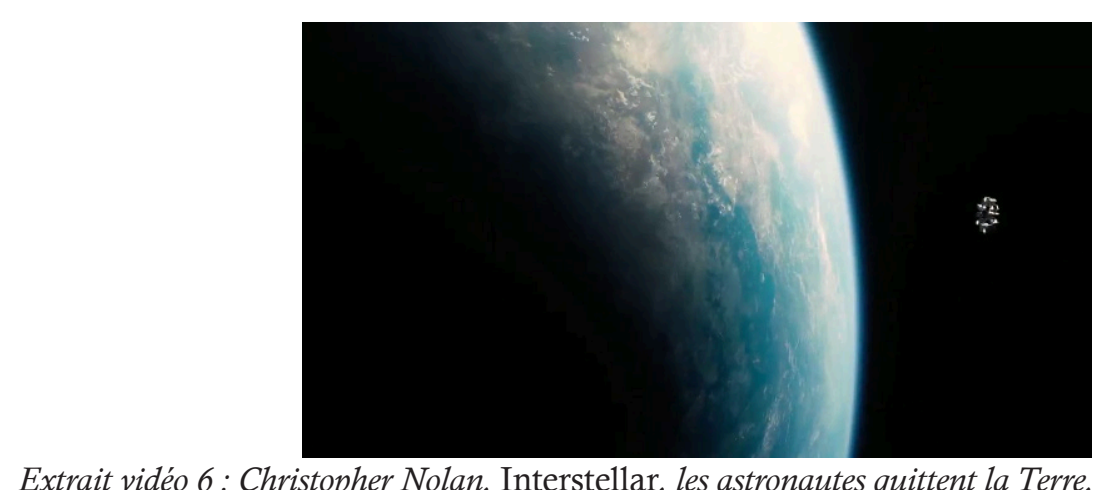

50:02-50:21 (C) Warner.

Un travail minutieux sur le mixage des différentes strates sonores

Loin de résulter des défauts techniques ou d'un manque de compétences, le parti-pris sonore de Nolan et Zimmer résulte de choix esthétiques et d'un travail minutieux sur le mixage des différentes strates. La séquence où Cooper tente d'amarrer son vaisseau à l'Endurance endommagée par une explosion accidentelle (extrait vidéo 7) est particulièrement remarquable à cet égard. Tout d'abord, la musique confère une urgence à la manœuvre de Cooper, tout en l'investissant d'un caractère grandiose en accord avec les choix de cadrage. L'accompagnement met en valeur la dimension à la fois cruciale et quasiment désespérée de la tentative de Cooper : il s'agit de la dernière chance pour Amelia et lui de survivre et de pouvoir revenir sur Terre. Par ailleurs, la musique fait écho aux mouvements visuels, dominés par les rotations, et à la composition plastique de l'image même où prévalent les cercles, avec ce vaisseau en « forme de roue rayonnée [qui] tourne sur elle-même " (Berthomieu 2014, p. 41).

Le cue «No Time for Caution», qui accompagne ces plans, repose en effet sur un motif circulaire au caractère hypnotique à l'orgue, dans la lignée des boucles orchestrales de Philip Glass ; il débute dans une nuance $f$ précisément au moment où Cooper annonce « Je m'amarre » à une Amelia abasourdie et terrifiée. L'amorce de l'ostinato apporte ainsi un élément de ponctuation significatif, d'une part, à la réplique déterminée de Cooper et, d'autre part, au plan rapproché sur Amelia mettant en valeur son changement immédiat d'expression et ses grands yeux écarquillés. Notons que la musique n'est jamais mixée en retrait par rapport au dialogue et aux effets sonores comme le veulent les conventions habituelles pour ce genre de scènes dans les blockbusters, qui relèvent traditionnellement d'un cinéma "voco-centriste» (Chion; Doane 1980, p. 52). L'orgue acquiert au contraire une présence submergeante dans toute la séquence. En outre, les ponctuations accentuées de percussions synthétiques s'inscrivent à mi-chemin 
entre la musique et le sound design et cohabitent parfaitement avec les bruitages à proprement parler. La puissance sonique qui résulte ici de l'écriture de Zimmer et du mixage est telle que de nombreux internautes ont qualifié cette séquence d'orgasme auditif (« eargasm»)!

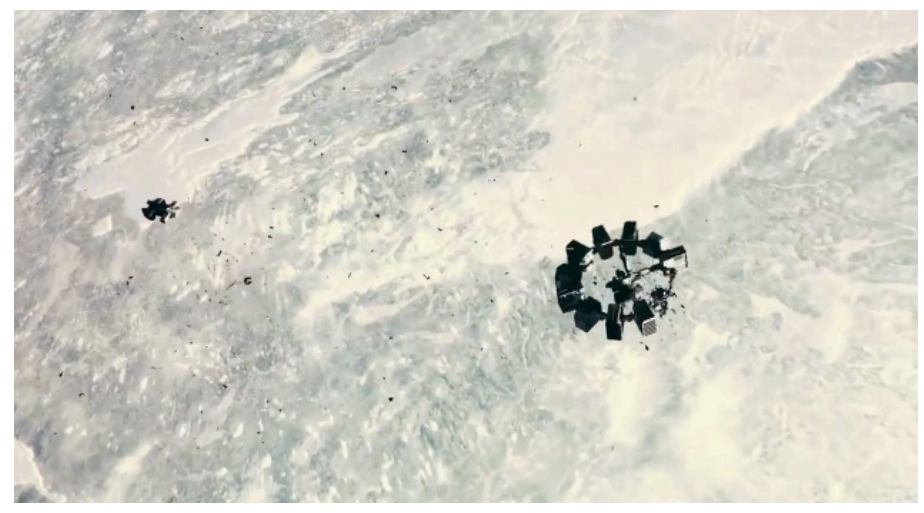

Extrait vidéo 7 : Christopher Nolan, Interstellar, Cooper tente une manoeuvre désespérée pour s'amarrer à l'Endurance, 02:07:45-02:09:46 (C) Warner.

Autre exemple emblématique de cette gestion minutieuse des différentes strates sonores, la scène où Cooper quitte sa famille met en œuvre un grand crescendo orchestral aboutissant à un tuilage saisissant entre la musique et les bruitages (extrait vidéo 8). Le motif des adieux, constitué de cinq notes, alterne dans toute la scène entre une version majeure fa-sib-la-sol-la et une présentation minorisée fa-sib-lab-sol-lab, entendues successivement lorsque Cooper démarre et quand Murph se précipite hors de la maison, rappelant en vain son père qui s'éloigne les yeux embués de larmes. Le volume de la musique augmente de façon continue, comme si elle ne parvenait plus à être contenue au vu de l'intensité poignante de la scène. Le mixage construit ainsi un parallèle entre les émotions ressenties par Cooper et la bande sonore, dans une forme de mixage empathique : la narration suit ici un crescendo émotionnel qui entraîne avec lui tous les éléments sonores - musique et mixage - vers une apothéose ${ }^{19}$.

La totalité de l'espace sonore est envahie par la musique, apportant ainsi une ampleur saisissante aux émotions des personnages jusqu'à un point de distorsion sonore, reflétant le drame de la séquence et le paroxysme émotionnel qui est en jeu. Le tuilage entre le climax musical et les sons de combustion de la fusée joue un rôle à la fois narratif et dramatique : l'arrachement tragique du héros à sa famille et à la Terre est symbolisé par cette saturation sonore et par cette fusion entre musique et bruitages. La scène (et l'ensemble du film) érige l'amour comme une force dont la puissance est égale à celle de la gravité. Le départ de Cooper est vécu comme une trahison par Murph, et la violence de cette séparation trouve une incarnation dans la puissance sonore de la séquence.

19 Nous tenons ici à remercier Luc Marcilly pour nous avoir suggéré cette notion de « mixage empathique » et pour son analyse sonore très intéressante de cette séquence. 


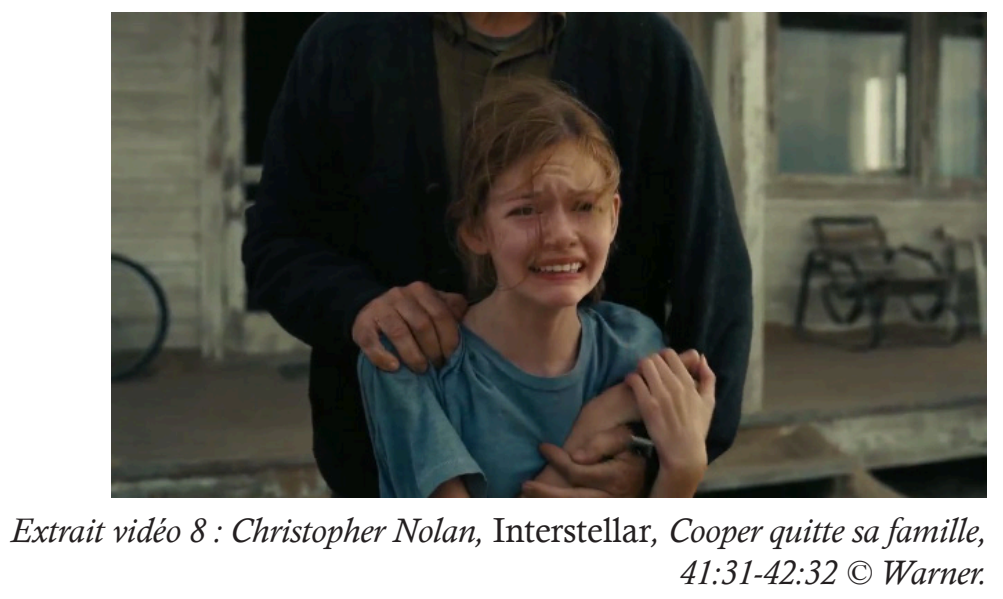

\section{CONCLUSION}

Fresque monumentale aux confins de l'espace, à la conquête de l'inconnu, Interstellar place l'amour et les liens humains au centre de sa narration. Comme le souligne Maxime Labrecque, l'amour reste

un substrat commun, une fibre vitale. [...] Après tout, comme nous l'avait enseigné Metropolis (Fritz Lang, 1927), le médiateur entre la main et le cerveau doit être le cœur. [...] Les personnages, malgré l'importance de leur mission, reviennent constamment à leurs préoccupations terrestres, à ceux qui sont restés là-bas (Labrecque 2015, p. 15).

De son côté, Pierre Berthomieu décèle dans le film de Nolan une ambition « d'inverser la froideur anempathique kubrickienne », en dramatisant " la réconciliation du cœur et du cerveau [dans un] essai de lyrisme héroïque » (Berthomieu 2014, p. 41).

La musique composée par Zimmer participe pleinement de cette focalisation sur l'humain et le drame familial. Elle offre un portrait mélancolique et intime des problématiques au cœur des relations humaines, de la réflexion proposée par le cinéaste autour des liens familiaux, de l'absence et du deuil : par un travail sur les timbres transparents et les sonorités épurées, par la création fréquente d'une temporalité suspendue, et par la composition d'un véritable thème d'amour, triste et délicat pour Cooper et Murph, l'accompagnement musical rehausse la valeur du sacrifice de Cooper, le caractère tragique des lignes temporelles parallèles pesant comme une malédiction sur la promesse du père faite à sa fille de rentrer sur Terre pour la retrouver. Les effets de plénitude sonique, à mi-chemin entre la musique et le sound design, accompagnent les moments les plus paroxystiques sur le plan émotionnel, revêtant ainsi un rôle narratif et dramatique crucial, loin des excès reprochés à la bande sonore d'Interstellar.

En filigrane, notre exploration audio-visuelle de l'un des films les plus marquants de la collaboration Nolan/Zimmer a aussi cherché à démontrer la diversité, la richesse et la flexibilité de l'approche zimmerienne de la musique de cinéma, souvent réduite à une mise en application schématique d'un certain nombre d'éléments stylistiques et de techniques d'écriture, indifféremment du film et de l'histoire concernés. Le modèle dégagé par la littérature existante nécessite d'être élargi et assoupli, afin de prendre 
en compte les multiples facettes de son écriture et surtout la créativité que nous avons tenté de mettre en valeur, aussi bien sur le plan de l'écriture, des rapports musique/ image, que des interactions entre l'accompagnement musical et le sound design. Les autres caractéristiques que nous proposons d'intégrer à l'approche analytique pourraient ainsi permettre de repenser la filmographie de Zimmer - y compris son versant épique - sous un angle riche et ouvert.

\section{BIBLIOGRAPHIE}

Berthomieu, Pierre (2004), La musique de film, Paris, Klincksieck.

Berthomieu, Pierre (2014), "Interstellar. Curiouser and Curiouser!", Positif, nº 646 (décembre), p. 40-41.

Berthomieu, Pierre (2013), "Splendeurs et misères du symphonisme hollywoodien, 1933-2012 », dans N. T. Binh (dir.), Musique \& cinéma. Le mariage du siècle ?, catalogue de l'exposition (Musée de la musique, Paris, 19 mars-18 août 2013), Paris, Actes Sud/Cité de la musique, p. 125-137.

Cathé, Philippe (2007), "Bruit et musique dans la course des Podracers de Star Wars, Episode I, The Fantom Menace (La Menace fantôme), 1999 », Musurgia, vol. XIV, n 2, p. 53-69.

Chion, Michel (s. d.), « 100 concepts pour penser et décrire le cinéma sonore», s. 1., http://michelchion. com/texts, consulté le 2 mars 2018.

De la Grange, Sophie (2002), «Zimmer, Hans », Positif, n 502 (décembre), p. 89.

Doane, Mary A. (1980), "Ideology and the Practice of Sound Editing and Mixing ", dans Teresa de Lauretis et Stephen Heath (dir.), The Cinematic Apparatus, New York, St Martin's Press, p. 4756.

Hexel, Vasco (2016), Hans Zimmer and James Newton Howard's The Dark Knight. A Film Score Guide, Lanham, Rowman \& Littlefield.

Hurwitz, Matt ([2007] 2012), "Sound for Picture: Hans Zimmer's Scoring Collective. Composer Collaboration at Remote Control Productions ", dans James Wierzbicki, Nathan Platte et Colin Roust (dir.), The Routledge Film Music Sourcebook, New York, Routledge, p. 254-257.

Huvet, Chloé (2015), "The Tudors de Trevor Morris : 1'importation du style "Media Ventures" dans une série télévisée ? ", dans Cécile Carayol et Jérôme Rossi (dir.), Musiques de séries télévisées, Rennes, Presses Universitaires de Rennes, p. 119-137.

Huvet, Chloé(2016), «La musicologie du cinéma : enjeux disciplinaires et problèmes méthodologiques», Intersections : revue canadienne de musique, vol. $36, \mathrm{n}^{\circ} 1,2016, \mathrm{p} .53-84$, https://www.muscan.org/en/ publications/intersections/952-intersections-vol-36-no-1.

Huvet, Chloé (à paraître), Composer pour l'image à l'ère numérique. Les deux trilogies Star Wars, Paris, Vrin.

Kilkenny, Katie (2014), «Why Interstellar's Organ Needs to Be So Loud», The Atlantic (11 novembre), https://www.theatlantic.com/entertainment/archive/2014/11/why-interstellars-organ-needs-tobe-so-loud/382619/, consulté le 23 mars 2017.

Labrecque, Maxime (2015), «Un peu plus haut, un peu plus loin. Interstellar », Séquences. La revue du cinéma, no 294 (janvier-février), p. 14-15.

Lauliac, Christian (1998), « Hans Zimmer », Positif, n 452 (octobre), p. 103.

Lehman, Frank (2016), «Manufacturing the Epic Score. Hans Zimmer and the Sounds of Significance», dans Stephen C. Meyer (dir.), Music in Epic Film. Listening to Spectacle, New York, Routledge, p. 27-55. 
Lowder, J. Bryan (2014), « Music of the Spheres. In Interstellar, Hans Zimmer Scores the Universe », Slate (novembre), http://www.slate.com/articles/arts/culturebox/2014/11/interstellar score hans zimmer composes music for the universe brilliantly.html, consulte le 24 mars 2017.

Macaulay, Sean (2013), « Scores that Make Good Movies Great », The Daily Telegraph (5 septembre), p. 24.

Nolan, Jonathan et Christopher (2014), Interstellar. The Complete Screenplay with Selected Storyboards, Londres, Faber \& Faber Limited.

Nolan, Christopher ([2014] 2015), Interstellar, 2 Blu-rays, Warner Home Video, 1000542383.

Reyland, Nicholas (2015), "Corporate Classicism and the Metaphysical Style ", Music, Sound, and the Moving Image, vol. 9, $\mathrm{n}^{\circ} 2$ (automne), p. 115-130.

Sobchack, Vivian (2005), "When the Ear Dreams. Dolby Digital and the Imagination of Sound ", Film Quarterly, vol. 58, no 4 (été), p. 2-15.

Tillman, Joakim (2016), "Topoi and Intertextuality. Narrative Function in Hans Zimmer's and Lisa Gerrard's Music to Gladiator » dans Stephen C. Meyer (dir.), Music in Epic Film. Listening to Spectacle, New York, Routledge, p. 59-85.

Wood, Aylish (2013), "Sonic Times in Watchmen and Inception", dans Carol Vernallis, Amy Herzog et John Richardson (dir.), The Oxford Handbook of Sound and Image in Digital Media, Oxford, Oxford University Press, p. 417-436.

Wright, Benjamin (2015), " Music and the Moving Image. A Case Study of Hans Zimmer ", dans John Shepherd et Kyle Devine (dir.), The Routledge Reader on the Sociology of Music, New York, Routledge, p. 319-327.

Zimmer, Hans (2014), Interstellar. Original Motion Picture Soundtrack, Sony Music Entertainment, 88875048122 . 